\title{
Atypical fungal thoracic vertebral osteomylitis resembling metastatic tumor and literature review of fungal vertebral osteomylitis
}

\section{Xifa Wu}

Zibo Central Hospital

Jian Sun

Weifang Medical University

Yanyan Li

Zibo Central Hospital

\section{Xueqiong Wu}

Zibo Central Hospital

\section{Zhen Wang}

Zibo Central Hospital

\section{Tao Li}

Zibo Central Hospital

Dexin Zou ( $\nabla 27981102 @ q q . c o m)$

Zibo Central Hospital https://orcid.org/0000-0002-3695-520X

\section{Case report}

Keywords: vertebral osteomyelitis, fungal, immunocompromised, atypical, metastatic tumor

Posted Date: October 1st, 2020

DOl: https://doi.org/10.21203/rs.3.rs-84001/v1

License: (9) This work is licensed under a Creative Commons Attribution 4.0 International License. Read Full License 


\section{Abstract}

\section{Case Description}

Fungal vertebral osteomylitis is a rare disease that difficult to diagnosis and cure. Because the early specific clinical manifestations of this disease are few and atypical, it is often missed or misdiagnosed, resulting in treatment delay and aggravation of the disease.

\section{Objective}

To report a rare case of cryptococcal osteomyelitis that resembles metastatic tumor happened in an immunecompromised patient with a history of mantle cell lymphoma and to review cases reported in literature.

\section{Study Design}

Based on imaging studies, metastatic tumor was highly suspected. For a further examination and therapy, the patient was referred to our spine clinic. After admission to our clinical, chest and abdominal CT were checked to evaluate the disease status.

\section{Treatments}

After general anesthesia, posterior thoracic pedicle screw fixation (T2.3.5.6) were performed, followed by posterior laminectomy and decompression of T4 vertebral body. The right facet joint of T3/4, costal transverse joint, right pedicle and transverse process of T4 vertebra were fully removed and curetted. After careful hemostasis, posterolateral bone grafting was performed. The nail-rod connection is fixed.

\section{Outcomes}

The postoperative intercostal neuralgia and chest pain were significantly relieved, without complications. The postoperative pathological results were suppurative inflammation with focal granuloma formation, dead bone formation and fungal (cryptococcal) infection.

\section{Conclusions}

When complicated with vertebral fungal spondylitis, no obvious abnormality and fever can be found. When pain or nerve dysfunction caused by spinal cord compression or nerve root compression occurs, surgical treatment can effectively relieve clinical symptoms.

\section{Case Description}

A 62-year-old female was admitted to our department because of a rise of back pain. She had a history of mantle cell lymphoma. She had been treated with chemotherapy for 2 years prior to admission. The Follow-up studies have not revealed any evidence of recurrence. About 2 months before visiting our clinic, 
her back pain developed. At that time, she visited local pain clinics and was treated with medication and injection. Her intercostal neuralgia aggravated during the period of conservative treatment. Then, she received thoracic magnetic resonance imaging (MRI) (Fig.1) and the result of the exam showed hypointense at T4 vertebral body, right pedicle and lamina in T1-weighted image. In addition, computed tomography (CT) showed osteolytic lesion in T4 right vertebral body, pedicle and facet joint. (Fig.1) Based on imaging studies, metastatic tumor was highly suspected. For a further examination and therapy, the patient was referred to our spine clinic.

\section{Treatment}

After admission to our clinical, chest and abdominal CT were checked to evaluate the disease status. There was no evidence of distant metastasis to internal organ or thorax. Lab examinations showed slight elevation of C-reactive protein (CRP) $(38.40 \mathrm{mg} / \mathrm{L}$; normal range, $0-5 \mathrm{mg} / \mathrm{L})$. However, antibody of

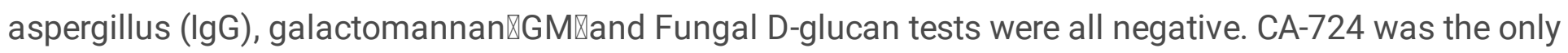
one tumor marker that mildly elevated $(47.93 \mathrm{ng} / \mathrm{ml}$, normal range, $0-6.9 \mathrm{ng} / \mathrm{ml})$. percentage of Lymphocyte and monocytes were $15.80 \%$ (normal rage,20-50) and $10.40 \%$ (normal rage $3-10$ ),

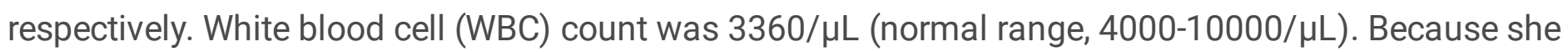
had a mantle cell lymphoma at this time, slight elevation of CRP was thought to be reasonable. In that period, her intercostal neuralgia aggravated and systemic symptom such as fever was not given enough attention. Because the patient has severe chest pain and intercostal neuralgia and is unable to sleep, he needs oral oxycodone tablets $40 \mathrm{mg}$, twice a day, as well as morphine injection to assist in analgesia. And her SINS score was about 9 , there was spinal instability, there were signs of surgical intervention, so we decided to give the patient surgical treatment.

The operation was performed with a posterior approach. First, posterior pedicle screw fixation was performed from T2.3 .5.6. Second, decompression was followed. T4 spinous process resection and right laminectomy were performed for decompression. Soft tissue invasion of right lamina, pedicle, right facet joint and right transverse facet joint were found during the operation. Fully scrape out the diseased tissue, saw rich blood supply, fully wash and implant allogeneic bone strips. Definitive pus-like discharge was not found, and soft material was identifiable in the center of T4 vertebral body. We obtained those materials and requested for pathologic examination. Before suturing the incision, we routinely washed the incision with saline more than $3000 \mathrm{ml}$.Postoperative administration of common antibiotics to prevent infection.

\section{Outcomes}

Final pathologic result showed small yeast-like organisms with Gomori methenamine silver, Fontana Masson, periodic acid-Schiff and Alcian blue stains (Fig.2). No tumor cells were shown by cytokeratin immunohisto chemical staining. Amphotericin B (50 mg/day) and fluconazole ( $800 \mathrm{mg} /$ day) were applied intravenously for about 1 week until disseminated cryptococcosis was ruled out. Then, fluconazole (450 mg/day) was applied orally for about 1 year. At the 3-months follow-up, back pain 
disappeared without any complications, and no specific abnormal findings were observed in plain radiographs (Fig.3).

\section{Discussion}

The incidence of fungal vertebral osteomyelitis has risen markedly in recent years because of several factors, primarily patient immunity and previous medical treatment. ${ }^{1,2}$ Slenker AK report that the most common factors for hematogenously spread candidal osteomyelitis infections are prior surgery, abdominal surgery in particular (60\%), at a noncontiguous site (44\%), broad spectrum antibiotics (41\%), intravenous drug abuse (26\%), central venous line insertion (26\%), immunosuppression (22\%), and total parenteral nutrition (TPN) (16\%). ${ }^{3}$ Prior surgical intervention (92\%) is the most common factor associated with the contiguous spread of infection causing fungal osteomyelitis, in particular prior spinal (21\%) or cardiothoracic surgery (52\%). Because presenting features can be as nonspecific as back or neck pain alone, fungal vertebral osteomyelitis can be difficult to recognize in its early stages. The rarity of the disease often leads to its misdiagnosis as a bacterial or tubercular infection. ${ }^{4,5}$ Appropriate treatment is often delayed, which can lead to poor outcomes. The outcomes of medical and surgical treatments are not always predictable. A gadolinium-enhanced MRI is still the gold standard for determining the lesion status, the treatment method, and the extent of surgery required. Computed tomography (CT imaging is valuable for biopsies, provides information about bone grafts and implants, and can be used as an alternative in patients for whom MRI is contraindicated. Fluorine-l8 fluorodeoxyglucose positron emission tomography (F-FDG-PET) is a relatively novel way to detect infection and has the highest diagnostic accuracy for diagnosing chronic osteomyelitis. ${ }^{1,2}$ As for the therapy for fungal osteomyelitis, If the diagnosis was before the endplate destruction, medical treatment can be the first option. If medical treatment failed and ESR and CRP levels were persistently elevated, the endplate had been destroyed at the initial presentation, the patient had neurological deficits, and epidural or paravertebral abscess accumulation, then surgical treatment is indicated for an open biopsy, decompression, and reconstruction. The surgical complication rate is high, and patients often need multiple surgeries to overcome the disease. However surgical treatment alone Is inadequate for FVO. Antifungal treatment often takes more than 3 months to lower ESR and CRP levels. ${ }^{1}$

The misdiagnosis and treatment delay of the patient in this case report should be responsible by the following $\rrbracket$

1. We have had very few patients with fungal spondylitis, so we lack the knowledge of diagnosis and differential diagnosis, especially in imaging.

2. The patient has a long history of halymphoma. The results of MRI examination before admission showed that the patient was highly suspected of spinal metastasis. However, in cases where laboratory and other imaging examinations did not support spinal metastases, we did not undergo a vertebral biopsy before open surgery. 
Although the incidence of fungal spondylitis has increased in recent years, due to the atypical of fungal osteomyelitis, it is still an infectious disease difficult to diagnose, identify and treat. In particular, its imaging features are often very similar to those of spinal tuberculosis, metastatic tumors, bacterial or tubercular infection. Therefore, strengthening the study of the imaging of fungal osteomylitis, familiar with the treatment and prevention of this disease is the key to accurate diagnosis and treatment.

\section{Conclusion}

When patients with lymphoma complicated with vertebral bone destruction, preoperative biopsy is needed to determine the nature of the lesion and guide the need for surgical treatment. When complicated with vertebral fungal spondylitis, the patient may not have fever, and no obvious abnormality can be found in laboratory examination. when pain or nerve dysfunction caused by spinal cord compression or nerve root compression occurs, surgical treatment is feasible, combined with postoperative drug treatment. can effectively relieve clinical symptoms. (1146 words)

\section{Declarations}

\section{Ethics approval and consent to participate}

The study involving human participants were reviewed and approved by Institutional Review Board of Zibo Central Hospital (No. 2018-1-96).

\section{Consent for publication}

The patients provided their written informed consent to participate in this study and publication.

\section{Availability of data and materials}

All datasets generated for this study are included in the article.

\section{Competing interest}

The authors declare that they have no conflict of interest.

\section{Funding}

Not application.

\section{Authors' contribution statements}

XW and DZ were responsible for initial design of this study. XW, JS, YL and TL were responsible for collection of clinical and radiologic materials. And XW, JS and ZW were responsible for writing the original manuscript. Finally, DZ was responsible for reviewing and revising this manuscript before submitting it. 


\section{Acknowledgement}

Not application.

\section{References}

1.DJ OG, Serletis D and Kazemi N. Fungal osteomyelitis with vertebral re-ossification. Int J Surg Case Rep 2016; 19: 1-3. 2015/12/23.

2.Shimizu J, Yoshimoto M, Takebayashi T, et al. Atypical fungal vertebral osteomyelitis in a tsunami survivor of the Great East Japan Earthquake. Spine (Phila Pa 1976) 2014; 39: E739-742. 2014/04/11.

3.Slenker AK, Keith SW and Horn DL. Two hundred and eleven cases of Candida osteomyelitis: 17 case reports and a review of the literature. Diagn Microbiol Infect Dis 2012; 73: 89-93. 2012/05/15.

4.McLeod N, Fisher M and Lasala PR. Vertebral osteomyelitis due to Candida species. Infection 2019; 47: 475-478. 2019/03/15.

5.Garbino J, Schnyder I, Lew D, et al. An unusual cause of vertebral osteomyelitis: Candida species. Scand $J$ Infect Dis 2003; 35: 288-291. 2003/07/04.

\section{Figures}

MRI
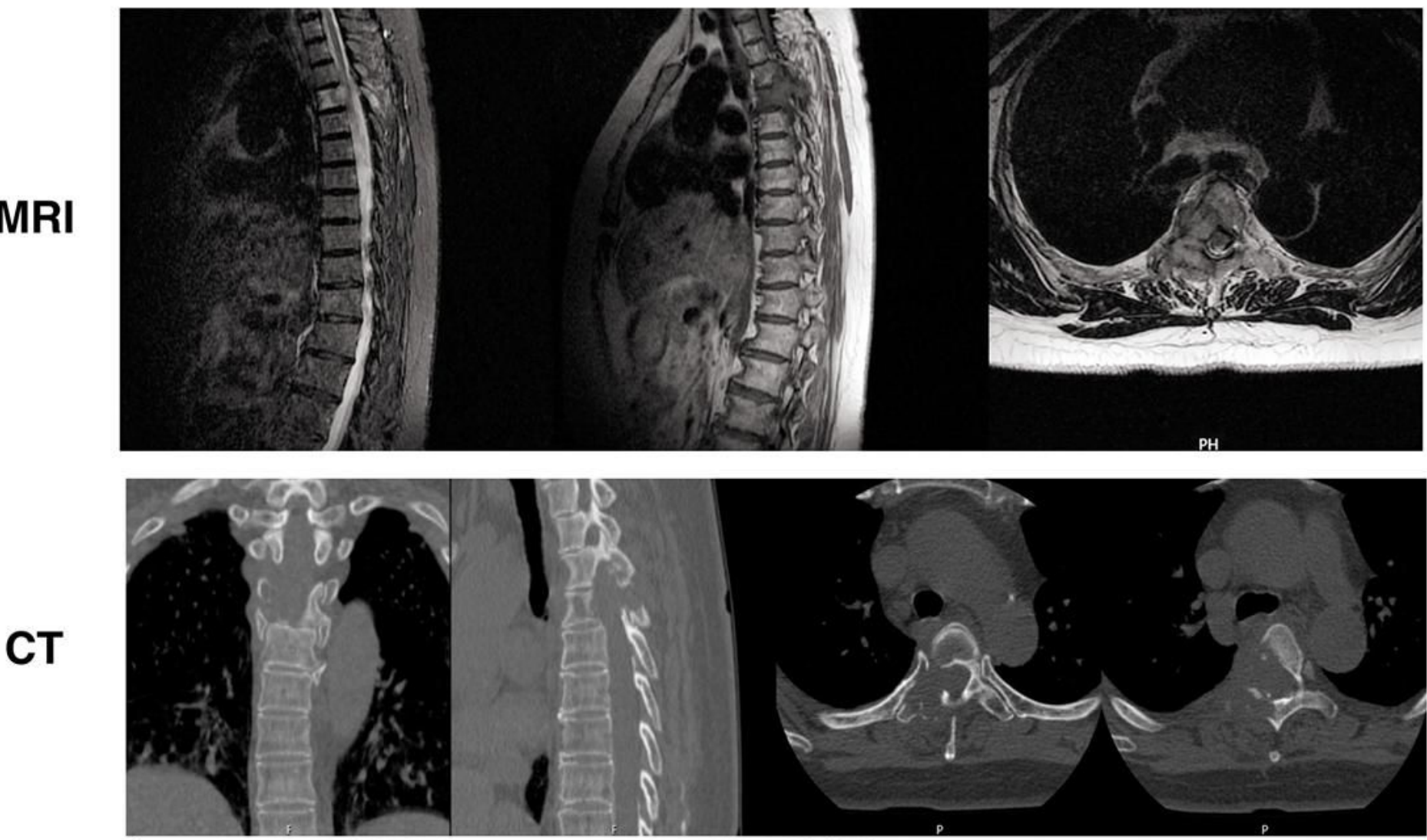


\section{Figure 1}

MRI pictures showed that Hypointense at T4 vertebral body, right pedicle and lamina in T1-weighted image. CT pictures showed that osteolytic lesion inT4 right vertebral body, Pedicle of vertebral arch and facet joint.

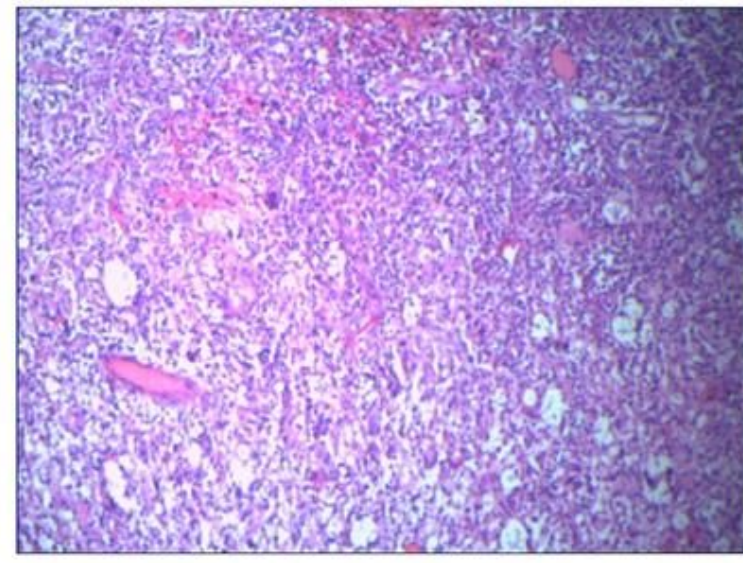

A
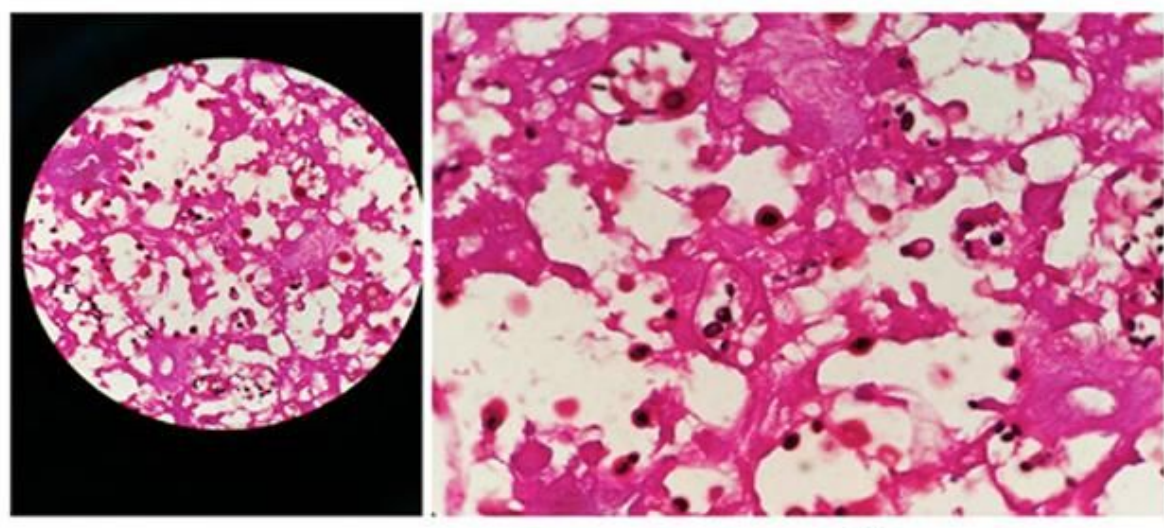

C

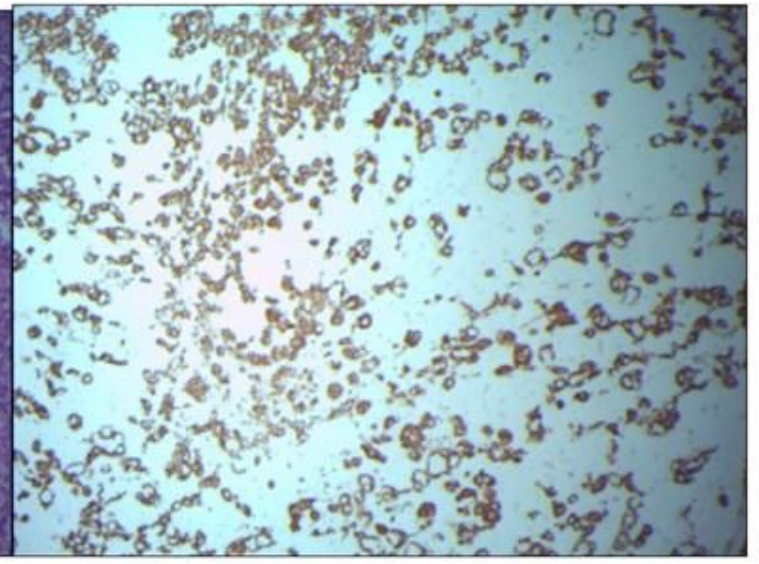

B

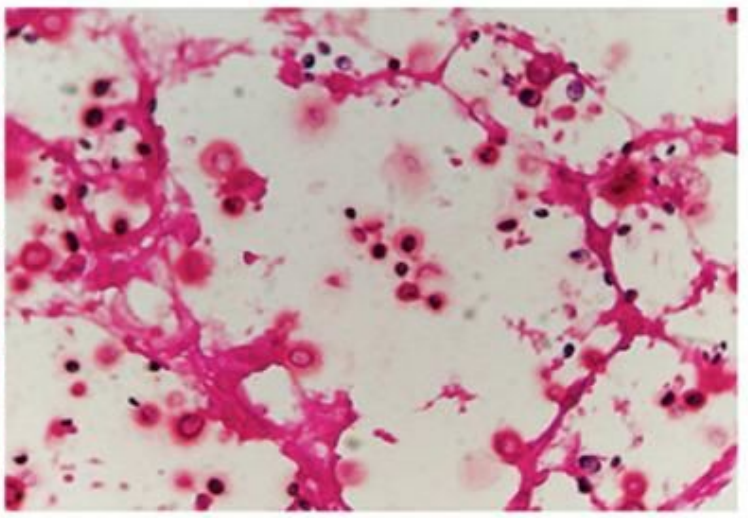

Figure 2

Tissue culture study after operation with different staining methods. Pathological Diagnosis $\bigotimes$ Diffuse nuclear detritus, infiltration of neutrophils, lymphocytes, plasma cells and multinucleated giant cell reaction were found in the intertrabecular and extraperiosteal fibrous adipose connective tissue of a small amount of bone tissue. Fibroblasts and capillaries proliferated, some trabecular calcium salts precipitated, and no osteocytes were found in the bone fossa. Combined with glycogen PAS positive, suppurative inflammation with focal abscess inflammatory granuloma formation, dead bone formation and fungal (Cryptococcus) infection were considered. A: hematoxylin-eosin staining $(H \& E)(\times 200)$; $B$ : immunohistochemical analysis $(\times 200)$ 

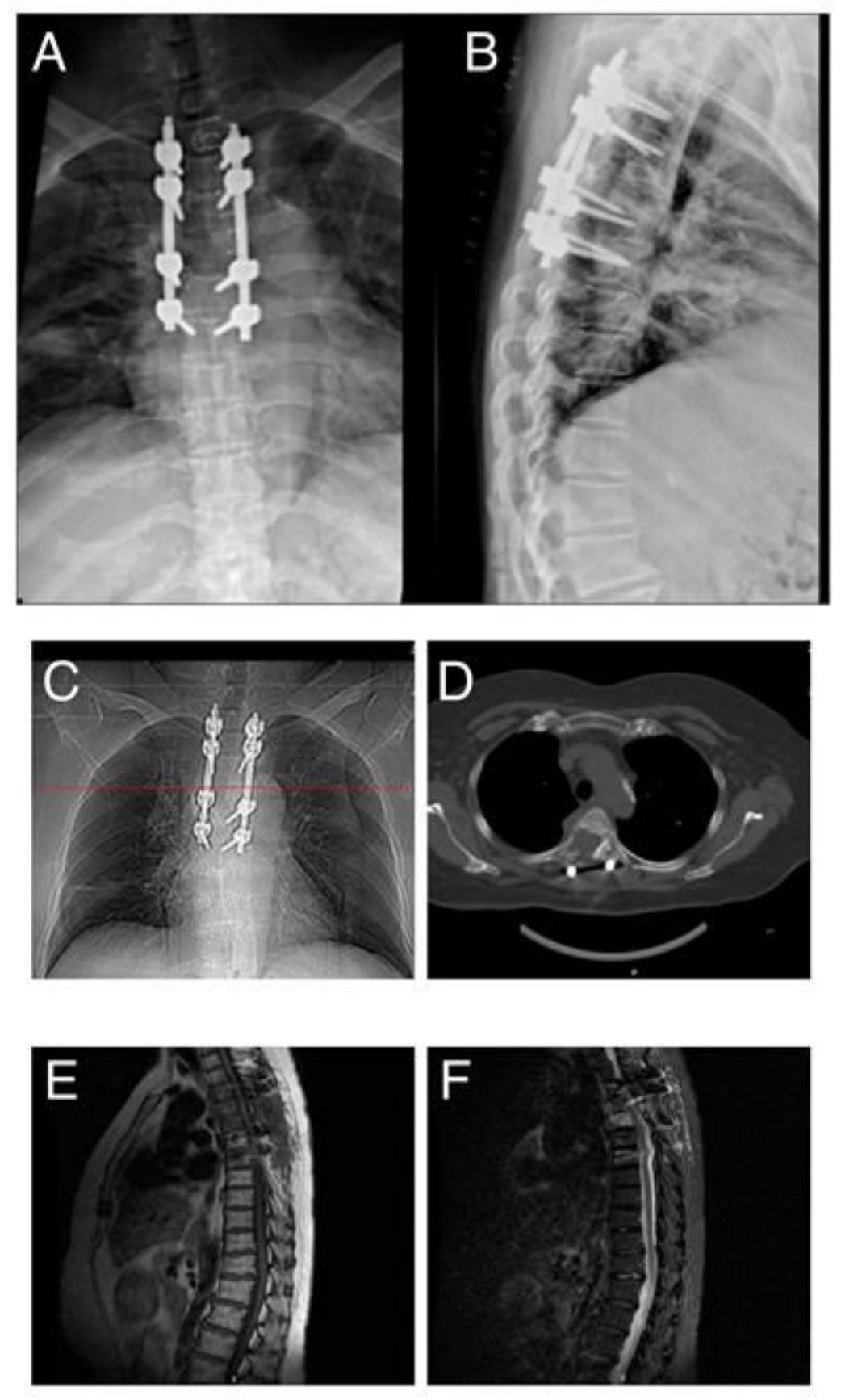

Figure 3

Radiographs 3 days after operation (A, B). The X-raxy (C), CT (D), MRI (E, F) pictures 3 months after operation. 